\title{
Silicone impressions vs photogrammetry (PicDental $@$ ) in dental prosthesis with multiple dental implants
}

IMPLANT THERAPY

OUTCOMES, PROSTHETIC

ASPECTS

Rodríguez Fernández, E. Vilariño Rodríguez, $R$.

Alfonso X el Sabio University, Madrid, Spain

\section{Abstract}

Abstract: The cad/cam is a design and manufacturing method that offers more accurate results and consistent fit than traditional methods ${ }^{1}$. The passive fit of structures retained by multiple implants has been and is a field of interest for the profession for many years: Although the definitive information on the adverse results related to the incorrect adjustment is scarce, the concern for the optimization of the adjustment has been expressed through different methods developed and applied to achieve better results ${ }^{2}$. In vitro studies indicate that there is not a manufacturing method providing absolute accuracy ${ }^{3}$. Therefore, methods can not ignore the clinical factors involved during the implant impression techniques. To resolve the contict, the photogrammetry establishes geometric relations between the image and he object at the pho images as an stl-file. The use of PicDentalß system allows to locate the precise $3-D$ position of implants to create an accurate cad/cam framework, providing accurate passive

Keywords: Dental implants, fit, cad/cam, photogrammetry impressions, dental prosthesis.

\section{Background and Aim}

Background: The procedure for making impressions of multiple implants is a challenge. Photogrammetry improves the results obtained than conventional impression techniques that are still employing silicones in situations when the number of implants involved is high. This improvement is reflected in a reduction of the working times consumed, the reduction of the radiological exposure times and the obtaining of a better passive adjustment

Aim: The purpose of this study is to evaluate the efficiency and effectiveness of the prosthetic fit obtained and work-time consumed by the impression techniques with silicones versus photogrammetry by PicDental®.

\section{Methods and Materials}
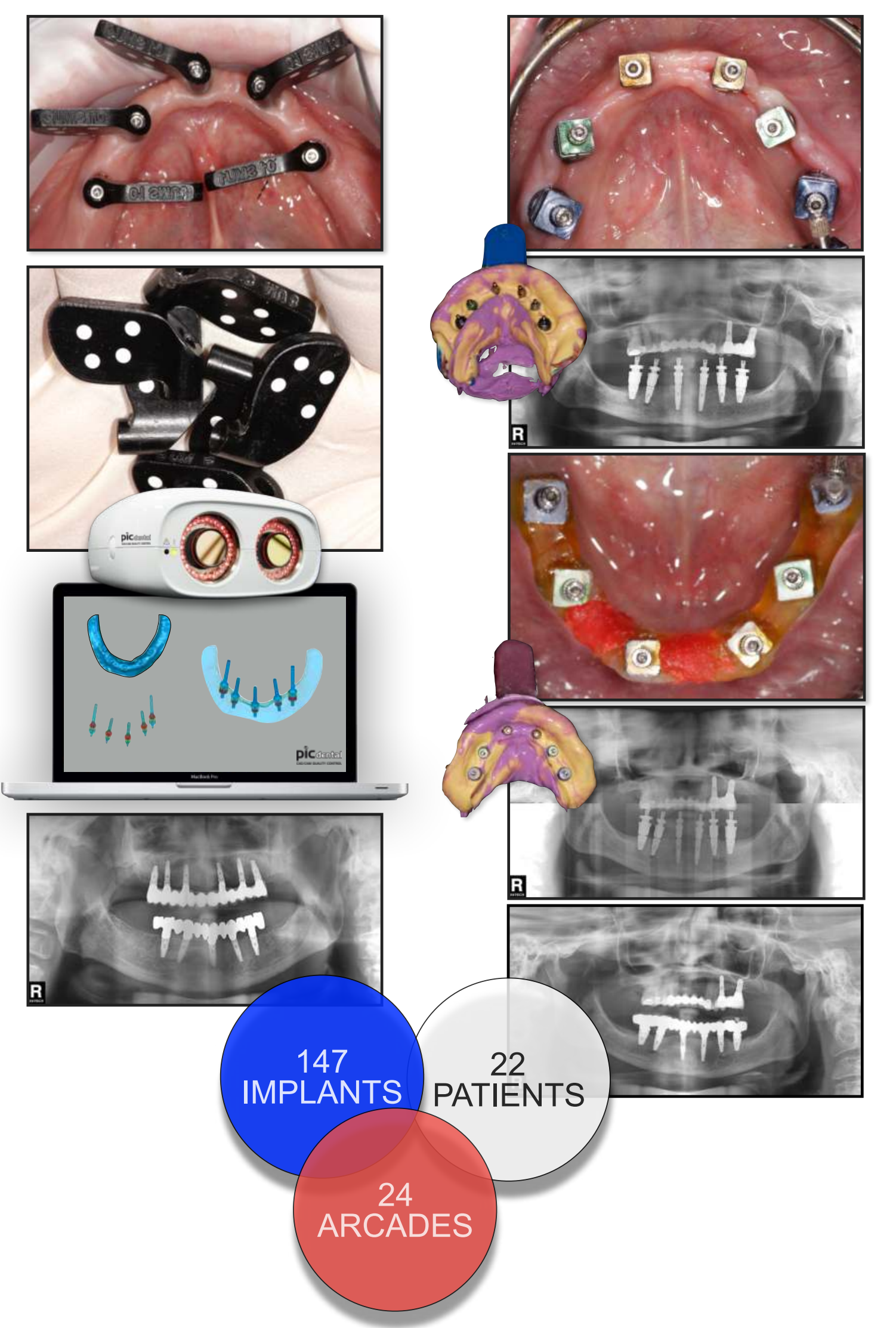

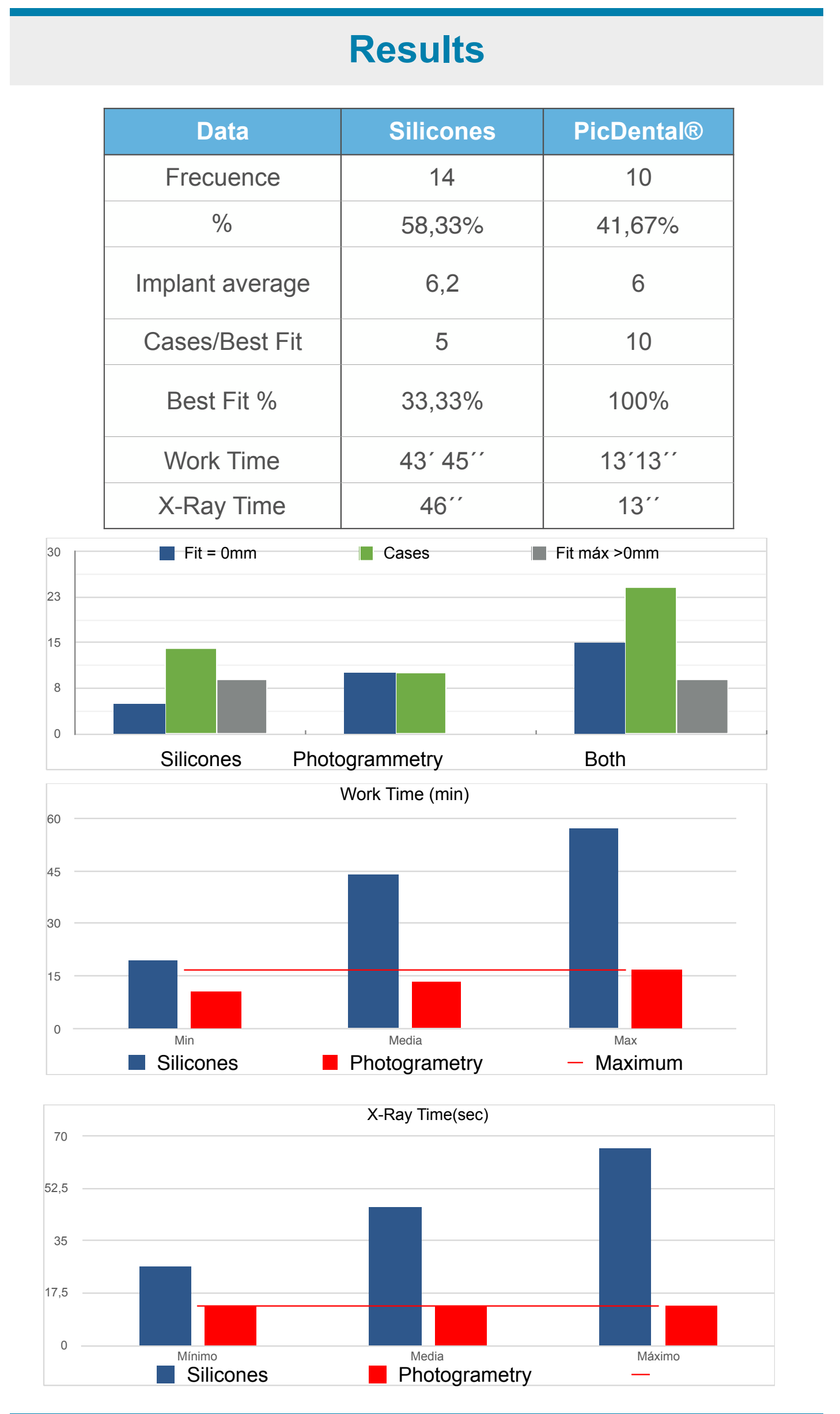

Conclusions

PicDental ${ }^{\circ}$ proves to be more effective and efficient procedure than custom open tray impression technique with silicones, when the number is equal to or greater than six units.

Digital workflows allow obtaining a high volume of information (inclination, distances between axes, depth, etc.) and relevant for the design and manufacture of a dental prosthesis.

Photogrammetry allows optimizing results and significantly reduces the working time.

PicDental ${ }^{\circledR}$ automatically save the obtained information and leaves to the dentists to interrupt the process if it will be necessary. In contrast, the silicones method forces to start from the beginning of the procedure in case of a setback.

Radiological exposure times revealed that X-ray consumption by the silicones maybe up to five times greater than PicDental $\AA$, which is less invasive.

\section{References}

1. Consensus statements and recommended clinical procedures regarding computer-assisted implant dentistry. The International journal of Oral \& Maxillofacial Implants. 2009; 24: 126-131.

2. Photogrammetry an alternative to conventional impressions in implant dentistry. A clinical pilot study. Int J Prosthodont. 1999;12: 363-368.

3. Photogrammetry and Conventional Impressions for Recording Implant Positions: A Comparative Laboratory Study. Clinical Implant Dentistry and Related Research. 2005; 1: 43-50.

4. An in vitro comparison of the photogrammetric and conventional complete arch implant impression techniques. J Prosthet Dent. 2013, 110:243-251.

5. Using stereophotogrammetric technology for obtaining intra-oral digital impressions of implants. JADA. 2014; 145: 338-344. 